\title{
INVESTIGAÇÃO DE FRAUDE EM SEBO BOVINO UTILIZADO PARA PRODUÇÃO DE BIODIESEL ATRAVÉS DA ESPECTROMETRIA DE MASSAS
}

\author{
N. V. REIS ${ }^{1}$, P. D. S. SANTOS ${ }^{2}$, N. B. MATTOS ${ }^{2}$, J. V. VISENTAINER ${ }^{2}$, O. O. SANTOS. \\ $\mathrm{JUNIOR}^{2}$ \\ ${ }^{1}$ Universidade Estadual de Maringá, Departamento de Engenharia Química \\ ${ }^{2}$ Universidade Estadual de Maringá, Departamento de Química \\ ${ }^{3}$ Universidade Estadual de Maringá, Centro de Ciências Agrárias \\ E-mail para contato: nvreis.eq@gmail.com
}

\begin{abstract}
RESUMO - A busca por fontes renováveis de energia lidera o novo século. Com isso, além da busca de novas tecnologias, abre-se também um olhar crítico voltado a evitar possíveis fraudes no fornecimento de matérias-primas, as quais podem acarretar inúmeros prejuízos no processo de produção de combustíveis, como a redução do rendimento de reação. $O$ trabalho em questão avalia a composição de diferentes sebos bovinos fornecidos à indústria de biodiesel a fim de confirmar se as matérias-primas recebidas pela indústria são de fato o que a empresa comprou. A partir de análises em cromatografia em fase gasosa e espectrometria de massas a fraude foi constatada utilizando marcadores lipídicos, visto que houve grande diferença na composição dos materiais.
\end{abstract}

\section{INTRODUÇÃO}

O crescente uso de automóveis e combustíveis gerou recentemente uma maior preocupação quanto a fontes renováveis de energia que não agridam o ambiente e também não se esgotem. Há uma grande preocupação na substituição das fontes de combustíveis fósseis, já que eles são finitos (Mahmudul et al., 2017). Atualmente, o petróleo é uma das sustentações da base energética do planeta. Ele é utilizado tanto na fabricação de diferentes combustíveis, como também no desenvolvimento de materiais e produtos, como os polímeros.

Dentre as buscas por substâncias alternativas ao petróleo, desenvolveu-se o biodiesel, que tem características e composição similares às do diesel, mas é proveniente de fontes renováveis, como óleos vegetais e animais (Mahmudul et al., 2017).

Dentre as matérias-primas utilizadas para a produção do biodiesel tem-se as provenientes de animais e vegetais. Algumas fontes vegetais de maior destaque são: soja, mamona, girassol, amendoim, algodão, dendê e pinhão-manso (Visentainer e Santos Júnior, 2013; Mahmudul et al., 2017). Um ponto positivo das fontes vegetais é que elas podem ser cultivadas, garantindo que a necessidade de matéria-prima seja suprida. Por outro lado, esta matéria-prima concorre diretamente com a produção de alimentos. Para evitar este problema, pode-se utilizar uma fonte animal, dentre as quais a mais utilizada é o sebo bovino, que possui 
o grande benefício do reaproveitamento, já que trata-se de um subproduto da indústria de alimentos.

O sebo bovino possui características específicas, como aspecto pastoso à temperatura ambiente e coloração esbranquiçada (Visentainer e Santos Júnior, 2013). Sua composição é dada principalmente por ácido oleico (45\%), ácido palmítico (30\%) e ácido esteárico (20$25 \%$ ). Seu baixo preço e alto teor de triglicerídeos são fatores decisivos na preferência por este tipo de matéria-prima. Com a ampla utilização do sebo bovino neste processo, há uma valorização deste produto. Com isso, é possível que hajam fraudes no fornecimento deste recurso com a adição de outros produtos, inclusive óleos residuais de fritura, óleos vegetais de menor valor, e outras gorduras animais provenientes de subprodutos de industrias que não possuam uma diferença muito grande na composição de ácidos graxos quando comparada ao sebo bovino. Isso faz com que a qualidade do sebo seja alterada.

Para a auxiliar na investigação de possíveis fraudes desta natureza, a cromatografia em fase gasosa acoplada a um detector de ionização em chama (GC-FID) é a tecnologia mais amplamente utilizada para a caracterização de materiais lipídicos (Fang et al., 2013). Neste tipo de análise, as amostras são derivatizadas, transformando os triglicerídeos em ésteres metílicos de ácidos graxos. A análise do equipamento gera picos de diferentes intensidades em diferentes tempos de retenção, que, quando comparados a um padrão, indicam a quantidade de cada éster metílico presente no material.

Quando comparamos apenas os ácidos graxos fica difícil a verificação de fraude, já que estes produtos adicionados têm percentual de ácidos graxos muito parecido com o sebo. Diversas pesquisas mostram que os triacilglicerois presentes nos alimentos funcionam como marcadores lipídicos, podendo então ser utilizados para detectar fraudes neste tipo de matériaprima. Para isso, a espectrometria de massas (GC/MS) (Fang et al., 2013; Lanças, 2009) é a técnica mais utilizada. A partir desta técnica, um "fingerprint" do material analisado é gerado através de uma varredura completa na razão massa/carga do material no intervalo desejado e o resultado é apresentado em menos de um minuto.

Desta forma, o objetivo do presente trabalho foi avaliar o perfil de triacilglicerois no sebo bovino recebido pela indústria BSBios, que produz biodiesel em Marialva-PR, e verificar a possível fraude nesta matéria-prima o utilizando-se das duas metodologias de análise apresentadas: a cromatografia em fase gasosa e a espectrometria de massas.

\section{MATERIAIS E MÉTODOS}

\subsection{Materiais}

Químicos: Metanol, ciclohexano, clorofórmio e etanol foram adquiridos da empresa Sigma-Aldrich (Darmstadt, Alemanha); éter etílico e isoctano foram adquiridos da empresa Nuclear e Anidrol (São Paulo, Brasil). Tricosanoato de metila (23:0) foi adquirido da empresa Sigma-Aldrich (Darmstadt, Alemanha). Para análise cromatográfica os reagentes utilizados foram de grau analítico, enquanto os solventes utilizados para a espectrometria de massas foi de grau HPLC. 
Sebo bovino: O sebo bovino analisado foi fornecido pela indústria de biodiesel BS Bios presente na cidade de Marialva - PR.

\subsection{Métodos}

Composição em ácidos graxos: De acordo com a ISO 5508 - "Analysis by gas chromatography of methyl esters of fatty acids" (1990).

Metilação: De acordo com ISO 5509 - "Preparation of methyl esters" (1978).

Parâmetros da cromatografia em fase gasosa: O sebo bovino padrão e o investigado foram analisados por cromatografia em fase gasosa, usando o equipamento Thermo Scientific CG acoplado ao detector de ionização em chama (FID), injetor split/splitless e coluna capilar de sílica fundida CP-7420 (Select FAME, $100 \mathrm{~m}$ de comprimento, 0,25 mm de diâmetro interno e $0,25 \mu \mathrm{m}$ de filme de cianopropil como fase estacionária). Parâmetros operacionais: temperatura da coluna de $165{ }^{\circ} \mathrm{C}$ por 18 minutos, aquecida a $235^{\circ} \mathrm{C}\left(4^{\circ} \mathrm{C} \cdot \mathrm{min}^{-1}\right)$ permanecendo por 20 minutos; temperaturas do injetor e detector foram mantidas a 230 e $250{ }^{\circ} \mathrm{C}$ respectivamente. Fluxos de gás foram de $1,2 \mathrm{~mL} \cdot \mathrm{min}^{-1}$ para o gás de arraste $\left(\mathrm{H}_{2}\right), 30 \mathrm{~mL} \cdot \mathrm{min}^{-1}$ para o gás de "make-up" $\left(\mathrm{N}_{2}\right)$ e no FID 30 e $300 \mathrm{~mL} \cdot \mathrm{min}^{-1}$ de gás $\left(\mathrm{H}_{2}\right)$ e ar sintético, respectivamente. As amostras foram injetadas no modo split com razão de 40:1. O volume injetado foi de $1,0 \mu \mathrm{L}$. A identificação e quantificação dos ácidos graxos foi feita por comparação entre a intensidade e os tempos de retenção com padrões interno e externo.

Quantificação dos ácidos graxos: Foi analisada de acordo com a área relativa percentual, de acordo com a regulamentação técnica de identidade e qualidade de óleos e gorduras (Visentainer e Santos Júnior, 2013), usando o software ChromQuest.

Determinação de triacilglicerois: triacilglicerol com amônio aduto $(+18.03 \mathrm{~m} / \mathrm{z})$ foi detectado pelo equipamento Waters XEVO TQD espectrômetro de massa electrospray com ionização operando em modo de íon positivo.

50,0 $\mu \mathrm{L}$ do sebo bovino sem modificações foi diluído em $950 \mu \mathrm{L}$ de clorofórmio. 5,0 $\mu \mathrm{L}$ desta solução foi misturado a 1,0 mL de uma solução 90:10 metanol/clorofórmio. 20,0 $\mu \mathrm{L}$ de uma solução 0,1 mol. $\mathrm{L}^{-1}$ de formiato de amônio foram adicionados à solução anterior. Esta solução final foi infundida a uma vazão de $10,0 \mu \mathrm{L} \cdot \mathrm{min}^{-1}$.

As voltagens do capilar e do cone foram de $3 \mathrm{kV}$ e $20 \mathrm{~V}$, respectivamente. ESI-MS foi focado nas faixas de massa de 100-1200 m/z. A temperatura de dessolvatação foi ajustada a $200{ }^{\circ} \mathrm{C}$. As diferentes espécies de TAG foram analisadas.

\section{RESULTADOS E DISCUSSÃO}

Visando verificar possíveis fraudes no sebo bovino adquirido, foi realizado primeiramente a análise da composição em ácidos graxos existentes na amostra padrão e na adquirida pela empresa BSBios, onde os resultados estão descritos na Tabela 1. 
Tabela 1 - Composição percentual dos ácidos graxos em sebos e valor de referência dos mesmos para esta matéria prima

\begin{tabular}{|c|c|c|c|}
\hline Ácidos Graxos & Sebo Padrão (\%) & Sebo Investigado (\%) & Valores de Referência (\%) \\
\hline \hline Ácido Mirístico (14:0) & $4,99 \pm 0,98$ & $1,02 \pm 1,11$ & $1,0-6,0$ \\
\hline Ácido Palmítico (16:0) & $29,26 \pm 1,93$ & $23,74 \pm 0,07$ & $20,0-37,0$ \\
\hline Ácido Palmitoleico (16:1) & $2,62 \pm 0,50$ & $0,51 \pm 0,07$ & $1,0-9,0$ \\
\hline Ácido Margárico (17:0) & $1,82 \pm 0,17$ & $5,33 \pm 0,16$ & $1,0-3,0$ \\
\hline Ácido Esteárico (18:0) & $24,78 \pm 3,47$ & $5,80 \pm 0,21$ & $25,0-40,0$ \\
\hline Ácido Oleico (C18:1) & $35,51 \pm 0,06$ & $35,84 \pm 0,42$ & $31,0-50,0$ \\
\hline Ácido Linoleico (C18:2) & $1,02 \pm 0,20$ & $27,75 \pm 0,30$ & $1,0-5,0$ \\
\hline
\end{tabular}

Observando os resultados dispostos na Tabela 1, o que mais se destaca é o baixo percentual de ácido esteárico e o elevado percentual de ácido linoleico para a amostra investigada quando comparada com a padrão e os valores de referência, o que vem explicar o aspecto líquido da amostra, enquanto o sebo tem aspecto pastoso a temperatura de $25^{\circ} \mathrm{C}$. Isto levanta a suspeita da amostra ter sido adulterada com algum óleo vegetal rico em ácido graxo linoleico, destes, o de menor custo é o óleo de soja.

As análises de ácidos graxos sozinhas apenas levantam suspeitas da adulteração, já a análise da amostra por espectrometria de massas tem o intuito de esclarecer melhor esta suspeita. Neste tipo de análise, os triacilglicerois encontrados em materiais lipídicos servem como "impressão digital" destas amostras, pois cada uma apresenta um perfil de triacilglicerois único. A Tabela 2 apresenta as massas dos triacilglicerois encontrados nos materiais analisados na faixa de interesse, de 800 a $950 \mathrm{~m} / \mathrm{s}$, enquanto as Figuras 1 e 2 apresentam os "fingerprints" do sebo padrão e do sebo investigado na mesma área de interesse, respectivamente.

Tabela 2 - Massas de triacilglicerois

\begin{tabular}{|c|c|c|c|}
\hline Triacilglicerol & Fórmula & $\mathrm{M} / \mathrm{S}$ & $\mathrm{M} / \mathrm{S}+\mathrm{NH}_{4}$ \\
\hline \hline LLL & $\mathrm{C}_{57} \mathrm{H}_{98} \mathrm{O}_{6}$ & 878 & 896 \\
\hline LLPo & $\mathrm{C}_{55} \mathrm{H}_{96} \mathrm{O}_{6}$ & 852 & 870 \\
\hline OLPo & $\mathrm{C}_{55} \mathrm{H}_{98} \mathrm{O}_{6}$ & 854 & 872 \\
\hline LLP & $\mathrm{C}_{55} \mathrm{H}_{98} \mathrm{O}_{6}$ & 854 & 872 \\
\hline PLPo & $\mathrm{C}_{53} \mathrm{H}_{96} \mathrm{O}_{6}$ & 828 & 846 \\
\hline OOL & $\mathrm{C}_{57} \mathrm{H}_{102} \mathrm{O}_{6}$ & 882 & 900 \\
\hline OOPo & $\mathrm{C}_{55} \mathrm{H}_{100} \mathrm{O}_{6}$ & 856 & 874 \\
\hline LOP & $\mathrm{C}_{55} \mathrm{H}_{100} \mathrm{O}_{6}$ & 856 & 874 \\
\hline POPo & $\mathrm{C}_{53} \mathrm{H}_{98} \mathrm{O}_{6}$ & 830 & 848 \\
\hline PLP & $\mathrm{C}_{53} \mathrm{H}_{98} \mathrm{O}_{6}$ & 830 & 848 \\
\hline PPPo & $\mathrm{C}_{51} \mathrm{H}_{96} \mathrm{O}_{6}$ & 804 & 822 \\
\hline OOO & $\mathrm{C}_{57} \mathrm{H}_{104} \mathrm{O}_{6}$ & 884 & 902 \\
\hline SOL & $\mathrm{C}_{57} \mathrm{H}_{104} \mathrm{O}_{6}$ & 884 & 902 \\
\hline OOP & $\mathrm{C}_{55} \mathrm{H}_{102} \mathrm{O}_{6}$ & 858 & 876 \\
\hline SLP & $\mathrm{C}_{55} \mathrm{H}_{102} \mathrm{O}_{6}$ & 858 & 876 \\
\hline POP & $\mathrm{C}_{53} \mathrm{H}_{100} \mathrm{O}_{6}$ & 832 & 850 \\
\hline SOO & $\mathrm{C}_{57} \mathrm{H}_{106} \mathrm{O}_{6}$ & 886 & 904 \\
\hline SLS & $\mathrm{C}_{57} \mathrm{H}_{106} \mathrm{O}_{6}$ & 884 & 902 \\
\hline SOP & $\mathrm{C}_{55} \mathrm{H}_{104} \mathrm{O}_{6}$ & 860 & 878 \\
\hline SOS & $\mathrm{C}_{57} \mathrm{H}_{108} \mathrm{O}_{6}$ & 888 & 906 \\
\hline PPP & $\mathrm{C}_{51} \mathrm{H}_{98} \mathrm{O}_{6}$ & 806 & 824 \\
\hline
\end{tabular}


Figura 1 - Triacilglicerois no sebo padrão.

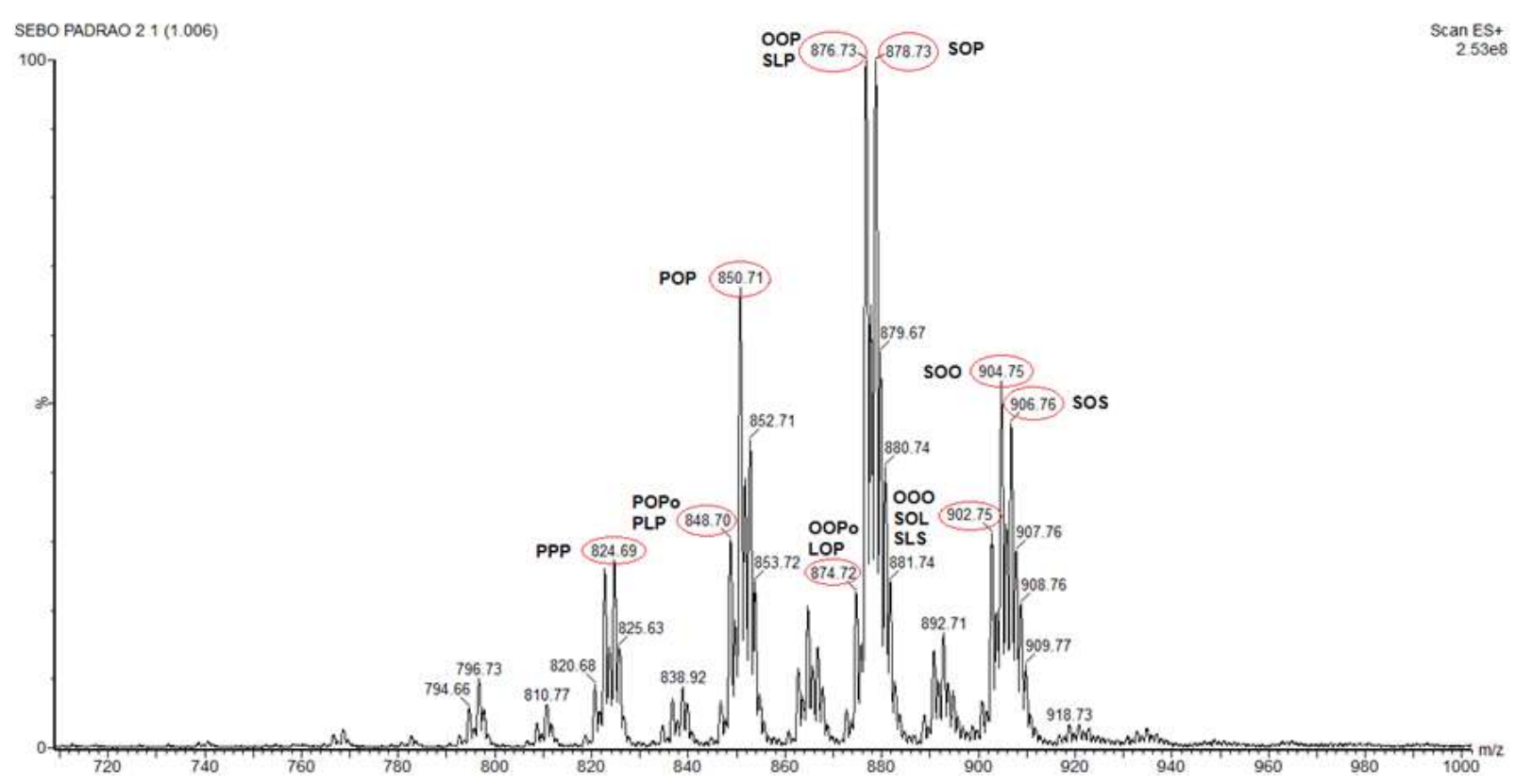

Figura 2 - Triacilglicerois no sebo investigado.

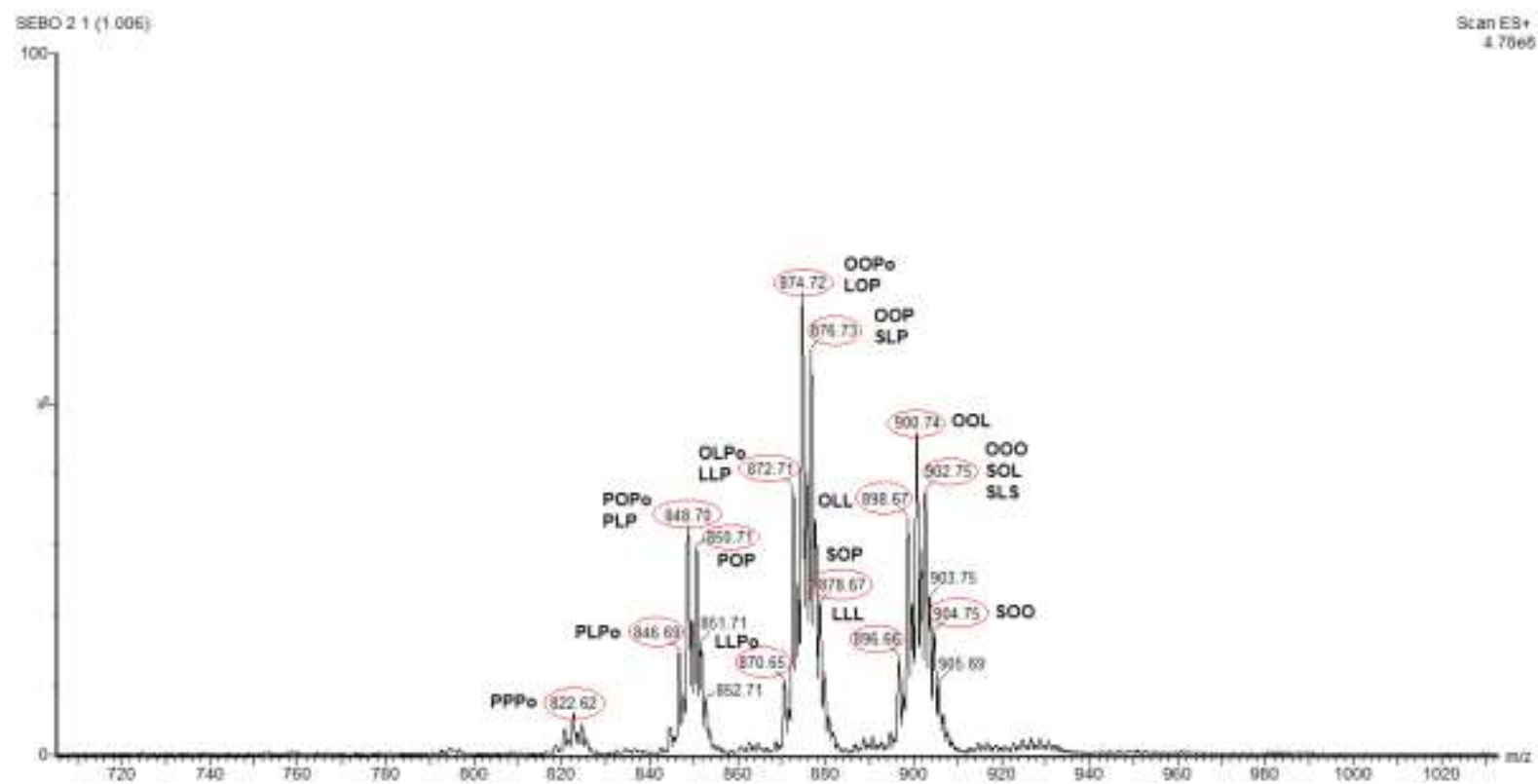

Por meio da Figura 1 é possível perceber a predominância de ácido esteárico (S), ácido oleico (O) e ácido palmítico $(\mathrm{P})$ no sebo bovino padrão, como também foi confirmado na Tabela 1. Já pela figura 2, nota-se que houve predominância dos ácidos oleico (O), palmítico (P) e também do linoleico (L) no sebo investigado, seguindo também a tendência mostrada pela Tabela 1. 
A presença de maior intensidade dos compostos de m/z 896 (LLL) e 898 (OLL) mostra que estes foram adulterados com óleo de soja, já que estes são os triacilglicerois de maior intensidade neste óleo, porém não estão presentes em elevadas intensidades na amostra de sebo padrão.

\section{CONCLUSÃO}

A partir dos estudos realizados pode-se perceber a necessidade de avaliar a matériaprima recebida para os processos de fabricação, garantindo assim o rendimento das reações e qualidade do produto final. A análise por espectrometria de massas mostrou-se uma boa técnica para a detecção de fraudes, por mostrar uma varredura bem detalhada sobre a amostra analisada. Além disso, a análise é rápida, exige pouca quantidade de amostra e pouco tratamento da amostra. Por fim, foi constatada a fraude devido ao perfil diferente apresentado pelas amostras e provavelmente tenha sido causada pela adição de óleo de soja, devido à presença de seus principais componentes, o LLL e o OLL, como já foi discutido.

\section{REFERENCIAS}

AGÊNCIA NACIONAL DO PETRÓLEO GÁS NATURAL E BIOCOMBUSTÍVEIS. (2016). Biodiesel. Disponível em: <http://www.anp.gov.br/wwwanp/biocombustiveis/biodiesel> Acesso em: 9 jan. 2017.

FANG, G.; GOH, J. Y.; TAY, M.; LAU, H. F.; LI, S. F. Y. Characterization of oils and fats by $1 \mathrm{H}$ NMR and GC/MS fingerprinting: Classification, prediction and detection of adulteration. Food Chemistry, v. 138(2-3), p. 1461-1469, 2013.

INTERNATIONAL ORGANIZATION OF STANDARIZATION - ISO 5508: Animal and vegetable fats and oils - Analysis by gas chromatography of methyl esters of fatty acids, 1990.

INTERNATIONAL ORGANIZATION OF STANDARIZATION - ISO 5509: Animal and vegetable fats and oils - Preparation of methyl esters of fatty acids, 1978.

LANÇAS, F. M. Cromatografia líquida moderna: HPLC/CLAE. Campinas: Editora Átomo, 2009.

MAHMUDUL, H. M.; HAGOS, F. Y.; MAMAT, R.; ABDUL ADAM, A.; ISHAK W. F. W.; ALENEZI, R. Production, characterization and performance of biodiesel as an alternative fuel in diesel engines - A review. Renewable and Sustainable Energy Reviews, v. 72, p. 497-509, 2017.

VISENTAINER, J. V.; SANTOS JÚNIOR, O. de O dos. Produção e Controle de Qualidade do Biodiesel de Óleos e Gorduras. Maringá: EDUEM, 2013. 